\title{
Ultrasonically Assisted Deposition of Colloidal Crystals
}

\author{
Sabine Wollmann, ${ }^{1,2, a)}$ Raj B. Patel, ${ }^{2}$ Achim Wixforth, ${ }^{1,3}$ and Hubert J. Krenner ${ }^{1,3}$ \\ ${ }^{1)}$ Lehrstuhl für Experimentalphysik 1 and Augsburg Centre for Innovative Technologies (ACIT), Universität Augsburg, \\ 86159 Augsburg, Germany \\ ${ }^{2)}$ Centre for Quantum Dynamics, Griffith University, Brisbane 4111, Australia \\ ${ }^{3)}$ Nanosystems Initiative Munich (NIM), Schellingstr. 4, 80779 München, Germany
}

(Dated: 14 July 2014)

Colloidal particles are a versatile physical system which have found uses across a range of applications such as the simulation of crystal kinetics, etch masks for fabrication, and the formation of photonic band-gap structures. Utilization of colloidal particles often requires a means to produce highly ordered, periodic structures. One approach is the use of surface acoustic waves (SAWs) to direct the self-assembly of colloidal particles. Previous demonstrations using standing SAWs were shown to be limited in terms of crystal size and dimensionality. Here we report a technique to improve the spatial alignment of colloidal particles using traveling SAWs. Through control of the radio frequency (RF) power which drives the SAW we demonstrate enhanced quality and dimensionality of the crystal growth. We show that this technique can be applied to a range of particle sizes in the $\mu \mathrm{m}$-regime and may hold potential for particles in the sub- $\mu \mathrm{m}$-regime.

Colloidal particles, and their ability to self-assemble, are a highly investigated research area by many groups worldwide. Colloidal particles dispersed in a medium can self-assemble due to their small size and their ability to move with respect to each other ${ }^{1}$. These can be used to create three-dimensional periodic structures for a broad range of applications such as templates for fabrication ${ }^{2}$, photonic crystals ${ }^{3}$, optical switches and filters ${ }^{4}$, chemical sensors ${ }^{5}$, transistors ${ }^{6}$, biological detectors ${ }^{7}$, or simulations of crystal kinetics ${ }^{8}$.

The underlying process for self-assembly relies on the balance of attractive and repulsive forces. The balance of the attractive van der Waals force and the repulsive electrostatic force, both of which are in the short range regime, stabilize the suspension ${ }^{1}$. The long range capillary force is responsible for the order of the colloidal particles. Capillary forces arise at the fluid interface between the particles and occur at the triple-phase boundary of colloidal particle, water and air. The attractive capillary interaction between the colloids exceeds the repulsive electrostatic forces by several orders of magnitude ${ }^{1}$. The capillary interaction between the particles is a manybody problem because of the multiple neighbours of each particle and the long range of the force ${ }^{1}$. Important parameters are the size and number of the particles in the suspension. Control of the evaporation rate results in a higher crystal quality with a reduced number of crystal defects $^{1}$. Long range hydrodynamic forces are caused by evaporation at the interface of the suspension and are the driving force for the crystal growth. The induced water flux to the phase boundary transports the colloidal particles to the nucleation site. The thickness of the water film at the meniscus, the number of arriving particles, and the shape of the meniscus determine the dimensionality of the crystal growth.

Van Blaaderen ${ }^{9}$ previously described the most common

\footnotetext{
a) Electronic mail: sabine.wollmann@griffithuni.edu.au
}

external forces for controlling the behaviour of colloids in dispersions. In this work the focus was on external fields which apply stress to gain control over the spatial alignment of the particles. Using confinements and gravity ${ }^{10}$ is also very common, however these methods offer limited control over the crystal parameters and are an inflexible steady system. Both long range forces and short range forces can be controlled by surface acoustic waves $(\mathrm{SAW})^{1,11,12}$. Interactions between the SAW and colloidal suspension result in (i) mixing of the suspension ${ }^{13}$, (ii) superposing of evaporation-induced water flux and Brownian motion $^{1}$, (iii) transfer of momentum ${ }^{14,15}$, (iv) deformation of the meniscus ${ }^{16}$ or $(\mathrm{v})$ transfer of heat and increase of evaporation rate ${ }^{14}$. Previous work ${ }^{11}$ demonstrated that ultrasound created by a piezoelectric transducer can be used to improve the structure of a selfassembled colloidal crystal. The compactness of the setup as well as the need for extended control over parameters like amplitude, frequency, and location of the waves, led to the use of SAWs. These traveling waves couple as a shear wave into the fluidic system. The interaction leads to the so-called 'acoustic streaming' 17,18 which can be controlled by the amplitude and frequency of the wave ${ }^{14}$. Wood et al. ${ }^{19,20}$ used counterpropagating SAWs to create standing waves which allow particles to align in a microfluidic system. They achieved a significant long range alignment in a two-dimensional array ${ }^{20}$. However, the size of the array was limited by the aperture of the transducer which generated the SAW. Moreover, control over the quality of the crystal structure was limited due to interactions between the colloidal particles and the static surface charge of the substrate ${ }^{20}$.

In this work, we present the directed self-assembly of colloidal particles by using acoustic streaming. The order of crystallinity and dimensionality can be chosen by the appropriate radio frequency $(\mathrm{RF})$ power for the selected particle size. This improves upon previous demonstrations because of their limitation in size and dimensionality of the colloidal crystal and lack of control over the quality of the spatial alignment ${ }^{20}$. 


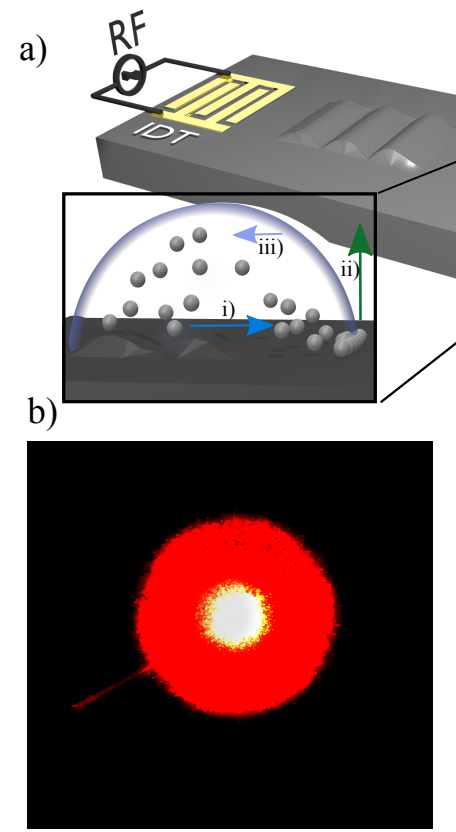

SAW off c)

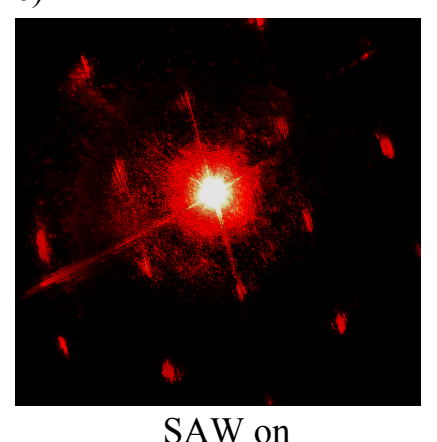

FIG. 1. a) Overview of the experimental setup. Interdigital transducers (IDT) create a surface acoustic wave (SAW) on the $\mathrm{LiNbO}_{3}$ substrate. The SAW travels towards the colloidal suspension where it couples into the fluid. The structure of the colloidal crystal is observed, after evaporation of the droplet, by optical diffraction using a laser beam with $\lambda=635 \mathrm{~nm}$. The inset shows the direction of the forces in the colloidal suspension. i) The direction of the hydrodynamic force, which transports particles to the nucleation site, ii) the direction of the water movement caused by iii) the evaporation at the triple-phase boundary. b) With no SAW applied there is no periodic structure visible in the diffraction pattern. c) Conversely, when the SAW is applied, assisted deposition takes place resulting in a hexagonal diffraction pattern with clear periodicity.

Colloidal solutions of polystyrene particles ${ }^{21}$ with a diameter of $3.00 \pm 0.07 \mu \mathrm{m}, \quad 2.00 \pm 0.03 \mu \mathrm{m}$ and $1.00 \pm 0.01 \mu \mathrm{m}$ were used in this work. For each size, a solution of particle concentration $8 \times 10^{2} \mu \mathrm{L}^{-1}$ was prepared. All experiments were performed using $1.5 \mu \mathrm{L}$ of solution. An overview of the setup used in this work is given in Fig. 1(a).

Metallic interdigital transducers (IDT) were fabricated on piezoelectric $17.5 \times 17.5 \mathrm{~mm} 128^{\circ}$-rot Y-cut Lithium niobate $\left(\mathrm{LiNbO}_{3}\right)$ wafers by photolithography. An adhesion layer of $10 \mathrm{~nm} \mathrm{Ti}$ was deposited followed by a conducting layer of $50 \mathrm{~nm} \mathrm{Al}$, the metallic structure was then covered by a capping layer of $\mathrm{SiO}_{2}$. This sputtered layer prevents the contact of water with the IDT. The center of the wafer remains free of $\mathrm{SiO}_{2}$ to allow the colloidal solution to interact directly with the $\mathrm{LiNbO}_{3}$ surface. The chip is packaged in a chip carrier to enable electrical connectivity. The electrically generated signal is an ultrafast agitated pulse with a carrier fre- quency of $152 \mathrm{MHz}$. A low duty cycle of $0.2 \%$ is used to reduce heat transfer to the microfluidic system. The power of the RF-signal is proportional to the square of the SAW amplitude. Applying an RF signal to the IDT creates a SAW directed towards the center of the substrate. The pulsed wave, which allows better control of the evaporation rate, interacts with the microfluidic system as described above ${ }^{14}$. The location and direction of the wave is responsible for the characteristic streaming profile $^{22}$ and depends on the frequency and amplitude of the $\mathrm{SAW}^{14}$. These parameters can be controlled by the positioning of the droplet, the IDT design, and the applied RF power. The SAW interacts with the complex colloidal forces. The inset of Fig. 1(a) shows the directions in which these forces act. The hydrodynamic force (i), directed towards the meniscus, is responsible for the particle transport to the nucleation site. It is dependent on the evaporation rate (ii) at the triple-phase boundary. At this water-air-substrate interface capillary forces occur and the crystal begins to grow towards the center of the droplet. The direction of the drying suspension is towards the centre of the droplet as indicated in (iii). To monitor the interactions between the SAW and the fluidic system, either an optical microscope or a crystal diffraction setup can be used. To observe the size and geometrical arrangement of the colloidal structure, the substrate was imaged using a bright-field transmitted microscope with a CCD camera (Hamamatsu C8484-05C). In addition, an optical diffraction setup (Fig. 1(a)) was used to examine the periodicity and geometry of the colloidal structure. The latter consists of a diode laser $(\lambda=635 \mathrm{~nm})$ and a CCD camera (Panasonic Tz10) mounted behind a screen where the pattern is projected when a laser is incident on the sample. The images and diffraction patterns were obtained after evaporation of the droplet for enhanced resolution.

Fig. 1(b) and (c) shows the diffraction pattern of the colloidal structure without and with an applied SAW during the deposition of the crystal. The images were obtained following complete evaporation of water from the solution, and deposition of the $3 \mu \mathrm{m}$ colloidal particles. Fig. 1(b) shows no periodic structure in the diffraction pattern when there is no SAW applied during the deposition. This indicates that neither short range nor long range order is present in the crystal. Though the complex forces in colloidal suspension are responsible for the self-assembly of the particles, it is a probabilistic process which needs a further external force to make it deterministic. The diffraction pattern of the SAW assisted deposition (Fig. 1(c)) shows a hexagonal pattern indicating a highly ordered crystal. The $60^{\circ}$ angle between the diffraction spots suggests a face-centered cubic (f.c.c.) crystal structure. The $60^{\circ}$ angle was also measured between the colloidal particles in the (111) lattice plane of the f.c.c. crystal structure. This arrangement maximizes the packing efficiency in the crystal unit cell and is therefore the most energetically favorable packing formation.

In acoustic streaming, the magnitude of the acoustic 


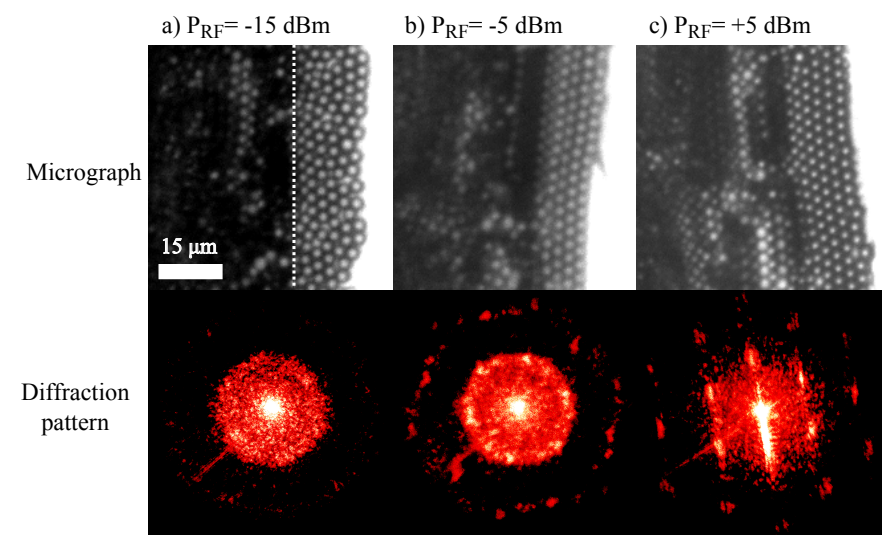

FIG. 2. Micrographs of the colloidal crystals of $3 \mu \mathrm{m}$ particles for input of (a) $-15 \mathrm{dBm}$, (b) $-5 \mathrm{dBm}$, and (c) $+5 \mathrm{dBm}$. The nucleation site along the meniscus is shown on the right side of the dotted line in (a). In (a) the corresponding diffraction patterns shows a Debye-Scherrer ring. With increasing power the first diffraction spots appear (b) followed by a hexagonal diffraction pattern with clear periodicity (c).

velocity field is linearly dependent on the RF power applied to the $\mathrm{IDT}^{22}$. This manipulates the interaction between the SAW and the complex colloidal forces and influences the structural outcome of the deposition process. Fig. 2 shows the micrographs and the corresponding diffraction patterns of crystals with $3 \mu \mathrm{m}$ particles for RF-powers of $-15,-5$, and $+5 \mathrm{dBm}$.

The micrographs show that the nucleation of the colloidal crystal begins at the water-air-substrate interface. The particles begin to align in a monolayer at the growth interface. For $\mathrm{P}_{R F}=-15 \mathrm{dBm}$ we observe that the short range order dominates the deposition at the nucleation site on the right side of the dotted line in Fig. 2(a). Its corresponding diffraction pattern shows a DebyeScherrer-ring. This diffraction pattern for polycrystalline structures occurs if short range order is present. Thus, it indicates a two-dimensional structure in which short range dominates over long range order. Increasing the signal power leads to higher order of the colloidal structure (Fig. 2(b)). The monolayer along the growth interface shows an increase in size as well as short range and long range order for $\mathrm{P}_{R F}=-5 \mathrm{dBm}$. Though the diffraction pattern still shows a ring, faint diffraction spots begin to appear along the ring. This indicates that not only the monolayer at the interface, but also the multilayers further away, have a higher periodicity than the previous crystal. According to the diffraction pattern, a further increase in the power to $\mathrm{P}_{R F}=+5 \mathrm{dBm}$ results in a complete transition of the crystal structure from polycrystalline to monocrystalline. The capillary driving forces for the nucleation at the water-air-substrate interface comprise the flotation force and immersion force. Both long range forces are caused by the surface tension of the liquid which tries to reduce the energy of the system by deforming the surface of the liquid between the particles $^{1}$. As these attractive forces exceed the repelling electrostatic forces between the particles by several orders of magnitude, they can be exploited for the selfassembly process. The strength of the capillary forces can be controlled by varying the wetting angle, evaporation rate and the surface tension of the liquid. The hydrodynamic force, induced by the evaporation at the meniscus of the droplet, is a particle-transporting water flux to the air-water-substrate interface. This phenomenon is independent of interacting SAWs and is a driving force for the crystal growth from the interface towards the center of the droplet. The number of particles, which are available for crystal growth at the nucleation site, determine the quality and dimension of the crystal. This can be subdivided into three regimes. (i) A low number of particles reaches the interface and a high spatial quality can be achieved. However, crystal growth is limited to two dimensions with respect to the evaporating water of the suspension. (ii) The number of particles surpass a critical number, and the crystal growth will be extended into the third dimension. (iii) The number of particles far exceeds the critical number, and the quality decreases as there will be leftover, unformed, crystals. Using SAWs, we have control over which of these three regimes crystal growth occurs in. Due to heat transfer from the SAW to the colloidal suspension, the evaporation rate increases. The hydrodynamic force is proportional to the evaporation, and is responsible for the transport of the particles to the nucleation site. In addition, the SAW induced streaming, which depends on the RF power, also transports colloidal particles to the interface. By manipulating the RF power, the crystal growth can be shifted between these regimes. In Fig. 2 we demonstrate that the crystal structure can be controlled between polycrystalline and monocrystalline with $\mathrm{P}_{R F}=+5 \mathrm{dBm}$. The modulated meniscus and the enhanced evaporation rate result in an enlarged monolayer at the nucleation site due to an increase of the capillary forces.

As demonstrated, we are able to manipulate the crystal structure from polycrystalline to monocrystalline by varying the power of the input RF signal. Fig. 3 shows that this transition can be extended to smaller particle sizes. We observe diffraction patterns for 3, 2, and $1 \mu \mathrm{m}$ bead sizes of a SAW assisted deposition, for three different RF powers. The diffraction pattern for the $3 \mu \mathrm{m}$ particles shows a Debye-Scherrer-ring for $\mathrm{P}_{R F}=-15 \mathrm{dBm}$ (Fig. 3(a)) which transitions to a hexagonal pattern with increasing power. The diffraction patterns of the two smaller particle sizes in Fig. 3(b) and (c) show a similar behavior with variation of the RF-power. However, the hexagonal diffraction spots appear for the $2 \mu \mathrm{m}$ particle size at $\mathrm{P}_{R F}=-5 \mathrm{dBm}$ and for the $1 \mu \mathrm{m}$ particle size at $\mathrm{P}_{R F}=+5 \mathrm{dBm}$. The diffraction patterns for the remaining RF powers show Debye-Scherrer-rings. We now discuss the mechanism behind these observations. To obtain a higher dimensional crystal structure, the deposition of the colloidal particles have to be addressed by choosing the appropriate RF-power. This becomes necessary as 


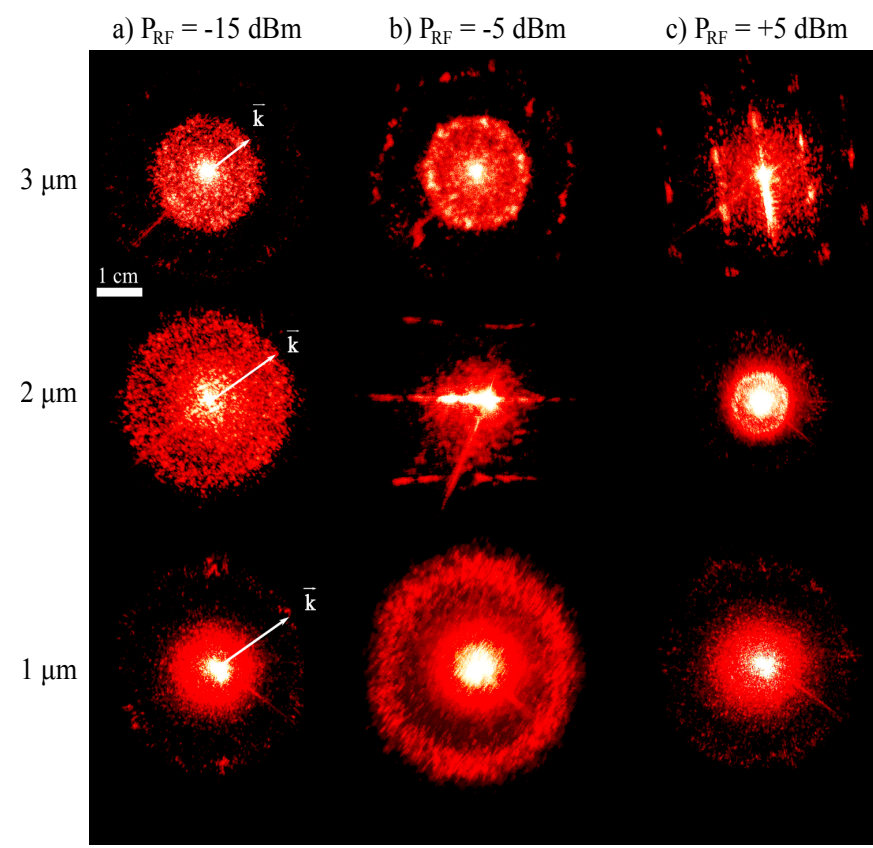

FIG. 3. The diffraction patterns for the particle sizes of 3, 2, and $1 \mu \mathrm{m}$ undergoing SAW assisted deposition with RF powers $-15,-5$ and $+5 \mathrm{dBm}$. The size of the diffraction pattern increases with decreasing particle size. a) The diffraction patterns for the $3 \mu \mathrm{m}$ particle size show a Debye-Scherrer ring which transitions to a hexagonal pattern with increasing RF power. b) The diffraction pattern for the $2 \mu \mathrm{m}$ shows a similar behavior, however less power is required to bring the colloidal particles into a crystalline state. c) For the smallest particle size of $1 \mu \mathrm{m}$, the lowest $\mathrm{RF}$ power of $\mathrm{P}_{R F}=-15 \mathrm{dBm}$ is sufficient to create a good spatial alignment.

some of the forces in the colloidal suspension depend on the particle size. The weak van der Waals forces are the interactions between the colloid and the substrate, as well as between the colloidal particles, these have a dependence on the particle size ${ }^{1}$. Moreover, the much stronger capillary force has a linear dependence with colloid size $\mathrm{e}^{23}$. The hydrodynamic force, which depends on the evaporation rate and the SAW induced acoustic streaming, is not related to the particle size. However, particles with lighter mass can be transported to the nucleation site in larger numbers. It is evident from Fig. 3 that we were able to influence the sensitive equilibrium between the number of particles at the nucleation site and the ordering force by varying the RF power. For the smaller particle sizes a lower RF power is required because of the alleviated transport to the meniscus. This dependence of the deposition on the $\mathrm{RF}$ power provides a readibly controllable parameter to manipulate the crystal structure for individual particle sizes.

In conclusion, we performed an experimental study on the ultrasonically assisted deposition of colloidal crystals by a SAW. First, we showed that applying a SAW supports the self-assembly of the colloidal particles. In addi- tion, we demonstrated that addressing the complex forces in the colloidal suspension by using different RF powers leads to control over the periodicity and growth of the crystal for a range of particle sizes. We find that our approach enables us to overcome limitations of previous work ${ }^{20}$. In particular, due to the control over the crystal quality with RF power, our approach holds potential for becoming a widely used method for fabrication of highly ordered three-dimensional colloidal crystals required for templates ${ }^{2}$, and may find use in a variety of applications $^{3-7}$. Furthermore, our setup is a versatile tool which allows such control without any changes in design from one particle size to another. The SAW is able to couple through a thin water film into an additional substrate and further to a colloidal suspension ${ }^{24}$. This makes the directed self-assembly independent of the substrate.

S.W. thanks Sergei Slussarenko for fruitful discussions. This work was supported by DFG via the Emmy Noether Program.

${ }^{1}$ Q. Li, U. Jonas, X. S. Zhao, and M. Kappl, A.-P. J. Chem. Eng. 3, 255 (2008).

${ }^{2}$ Y. A. Vlasov, X.-Z. Bo, J. C. Sturm, and D. J. Norris, Nature 414, 289 (2001), 10.1038/35104529.

${ }^{3}$ J. D. Joannopoulos, P. R. Villeneuve, and S. Fan, Nature 386, 143 (1997).

${ }^{4}$ G. Pan, R. Kesavamoorthy, and S. A. Asher, Phys. Rev. Lett. 78, 3860 (1997).

${ }^{5}$ J. H. Holtz and S. A. Asher, Nature 389, 829 (1997).

${ }^{6}$ D. V. Talapin and C. B. Murray, Science 310, 86 (2005).

${ }^{7}$ P. Alivisatos, Nat. Biotech. 22, 47 (2004), 10.1038/nbt927.

${ }^{8}$ H. J. Schöpe, G. Bryant, and W. van Megen, Phys. Rev. Lett. 96, 175701 (2006).

${ }^{9}$ A. van Blaaderen, MRS Bulletin 29, 85 (2004).

${ }^{10}$ M. Egen, R. Voss, B. Griesebock, R. Zentel, S. Romanov, and C. S. Torres, Chem. Mat. 15, 3786 (2003).

${ }^{11}$ O. D. Velev, A. M. Lenhoff, and E. W. Kaler, Science 287, 2240 (2000).

${ }^{12}$ J. Friend and L. Y. Yeo, Rev. of Mod. Phys. 83, 647 (2011).

${ }^{13}$ R. Shilton, M. K. Tan, L. Y. Yeo, and J. R. Friend, J. Appl. Phys. 104, (2008).

${ }^{14}$ C. J. Strobl, A. Rathgeber, A. Wixforth, C. Gauer, and J. Scriba, in Ultrasonics Symposium, 2002. Proceedings. 2002 IEEE, Vol. 1 (2002) pp. 255-258.

${ }^{15}$ S. Shiokawa, Y. Matsui, and T. Moriizumi, Jap. J.of Appl. Phys. 28, 126 (1989).

${ }^{16}$ A. Wixforth, C. Strobl, C. Gauer, A. Toegl, J. Scriba, and Z. Guttenberg, Anal. Bioanal. Chem. 379, 982 (2004).

${ }^{17}$ W. L. M. Nyborg, Acoustic streaming, Physical Acoustics: Principles and Methods, Vol. Voll II B (Academic Press, New York, 1965).

${ }^{18}$ T. M. Squires and S. R. Quake, Rev. of Mod. Phys. 77, 977 (2005).

${ }^{19}$ C. D. Wood, S. D. Evans, J. E. Cunningham, R. O'Rorke, C. Wlti, and A. G. Davies, Appl. Phys. Lett. 92, (2008).

${ }^{20}$ C. D. Wood, J. E. Cunningham, R. O'Rorke, C. Wlti, E. H. Linfield, A. G. Davies, and S. D. Evans, Appl. Phys. Lett. 94, (2009).

${ }^{21}$ Supplied by Polysciences Inc.

${ }^{22}$ T. Frommelt, D. Gogel, M. Kostur, P. Talkner, P. Hanggi, and A. Wixforth, Ultrasonics, Ferroelectrics and Frequency Control, IEEE Transactions on 55, 2298 (2008).

${ }^{23}$ N. Denkov, O. Velev, P. Kralchevski, I. Ivanov, H. Yoshimura, and K. Nagayama, Langmuir 8, 3183 (1992).

${ }^{24}$ R. P. Hodgson, M. Tan, L. Yeo, and J. Friend, Appl. Phys. Lett. 94, 4 (2009). 